\title{
Origin of electron-hole asymmetry in the scanning tunneling spectrum of $\mathrm{Bi}_{2} \mathrm{Sr}_{2} \mathrm{CaCu}_{2} \mathrm{O}_{8+\delta}$
}

\author{
Jouko Nieminen ${ }^{1,2}$ * Hsin Lin $^{2}$, R.S. Markiewicz ${ }^{2}$, and A. Bansil ${ }^{2}$ \\ ${ }^{1}$ Institute of Physics, Tampere University of Technology, P.O. Box 692, 33101 Tampere, Finland and \\ ${ }^{2}$ Physics Department, Northeastern University, Boston, Massachusetts 02115
}

(Dated: Version of October 25, 2018)

\begin{abstract}
We have developed a material specific theoretical framework for modelling scanning tunneling spectroscopy (STS) of high temperature superconducting materials in the normal as well as the superconducting state. Results for $\mathrm{Bi}_{2} \mathrm{Sr}_{2} \mathrm{CaCu}_{2} \mathrm{O}_{8+\delta}$ (Bi2212) show clearly that the tunneling process strongly modifies the STS spectrum from the local density of states (LDOS) of the $d_{x^{2}-y^{2}}$ orbital of $\mathrm{Cu}$. The dominant tunneling channel to the surface Bi involves the $d_{x^{2}-y^{2}}$ orbitals of the four neighbouring $\mathrm{Cu}$ atoms. In accord with experimental observations, the computed spectrum displays a remarkable asymmetry between the processes of electron injection and extraction, which arises from contributions of $\mathrm{Cu} d_{z^{2}}$ and other orbitals to the tunneling current.
\end{abstract}

PACS numbers: 68.37.Ef 71.20.-b 74.50.+r 74.72.-h

Scanning tunneling spectroscopy (STS) has entered the realm of high-temperature superconductors powerfully by offering atomic scale spatial resolution in combination with high energy resolution. The physics of these materials is dominated by the cuprate layers, which are usually not exposed to the tip of the apparatus. Much of the existing interpretation of the spectra is based however on the assumption that the STS spectrum is directly proportional to the LDOS of the $\mathrm{CuO}_{2}$ layer, neglecting the effects of the tunneling process in modifying the spectrum in the presence of the insulating overlayers. Here, we focus on the Bi2212 system, which has been the subject of an overwhelming amount of experimental work 1[5, although our results bear more generally on the STS spectra of the cuprates.

Our analysis takes into account the fact that the current originating in the $\mathrm{CuO}_{2}$ layers reaches the tip after being 'filtered' through the overlayers of $\mathrm{SrO}$ and $\mathrm{BiO}$, and shows that instead of being a simple reflection of LDOS of the $\mathrm{CuO}_{2}$ layers, the STS signal represents a complex mapping of the electronic structure of the system. In particular, we find that the spectrum develops a striking asymmetry between positive and negative biases because $d_{z^{2}}$ and other orbitals begin to contribute to the tunneling current with increasing bias voltage. Although this asymmetry has often been taken to be the hallmark of strong correlation effects [6], our results indicate that the nature of the tunneling process itself induces significant electron-hole asymmetry even within the conventional picture, so that strong correlation effects on the STS spectrum will be more subtle than has been realized so far.

In order to construct a realistic framework capable of describing the STS spectrum of the normal as well as the superconducting state, we start with the normal state Hamiltonian

$$
\hat{H}_{1}=\sum_{\alpha \beta \sigma}\left[\varepsilon_{\alpha} c_{\alpha \sigma}^{\dagger} c_{\alpha \sigma}+V_{\alpha \beta} c_{\alpha \sigma}^{\dagger} c_{\beta \sigma}\right]
$$

\section{(a) Side view}

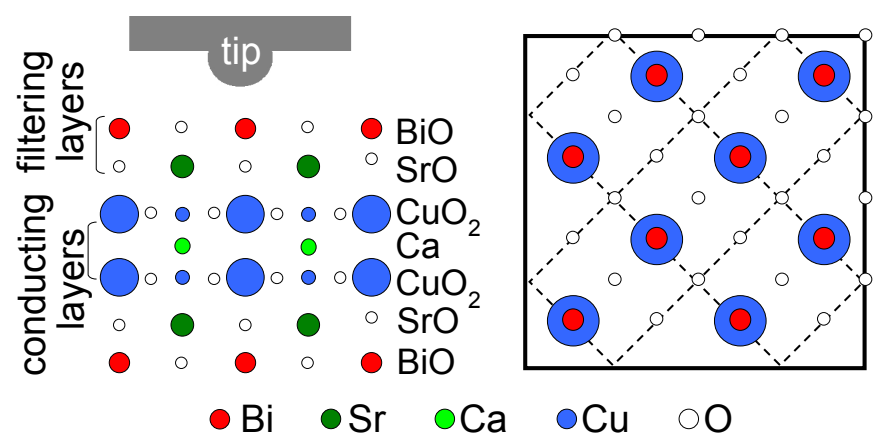

FIG. 1. (a) Side view of tip placed schematically on top of seven layers used to compute the tunneling spectrum of Bi2212, where the surface terminates in the BiO layer. Tunneling signal from the conducting $\mathrm{CuO}_{2}$ layers reaches the tip after passing through $\mathrm{SrO}$ and $\mathrm{BiO}$ layers. (b) Top view of the surface showing arrangement of various atoms. Eight two-dimensional real space primitive unit cells used in the computations are marked by dashed lines.

which describes a system of tight-binding orbitals created (or annihilated) via the real-space operators $c_{\alpha \sigma}^{\dagger}$ (or $c_{\alpha \sigma}$ ). Here $\alpha$ is a composite index denoting both the type of orbital (e.g. $\mathrm{Cu} d_{x^{2}-y^{2}}$ ) and the site on which this orbital is placed, and $\sigma$ is the spin index. $\epsilon_{\alpha}$ is the on-site energy of the $\alpha^{\text {th }}$ orbital. $\alpha$ and $\beta$ orbitals interact with each other through the potential $V_{\alpha \beta}$ to create the energy eigenstates of the system.

Superconductivity is included by adding a pairing interaction term $\Delta$ in the Hamiltonian:

$$
\hat{H}=\hat{H}_{1}+\sum_{\alpha \beta \sigma}\left[\Delta_{\alpha \beta} c_{\alpha \sigma}^{\dagger} c_{\beta-\sigma}^{\dagger}+\Delta_{\beta \alpha}^{\dagger} c_{\beta-\sigma} c_{\alpha \sigma}\right]
$$

We take $\Delta$ to be non-zero only between $d_{x^{2}-y^{2}}$ orbitals of the nearest neighbor $\mathrm{Cu}$ atoms, and to possess a d-wave form, i.e. $\Delta$ is given in momentum space by 


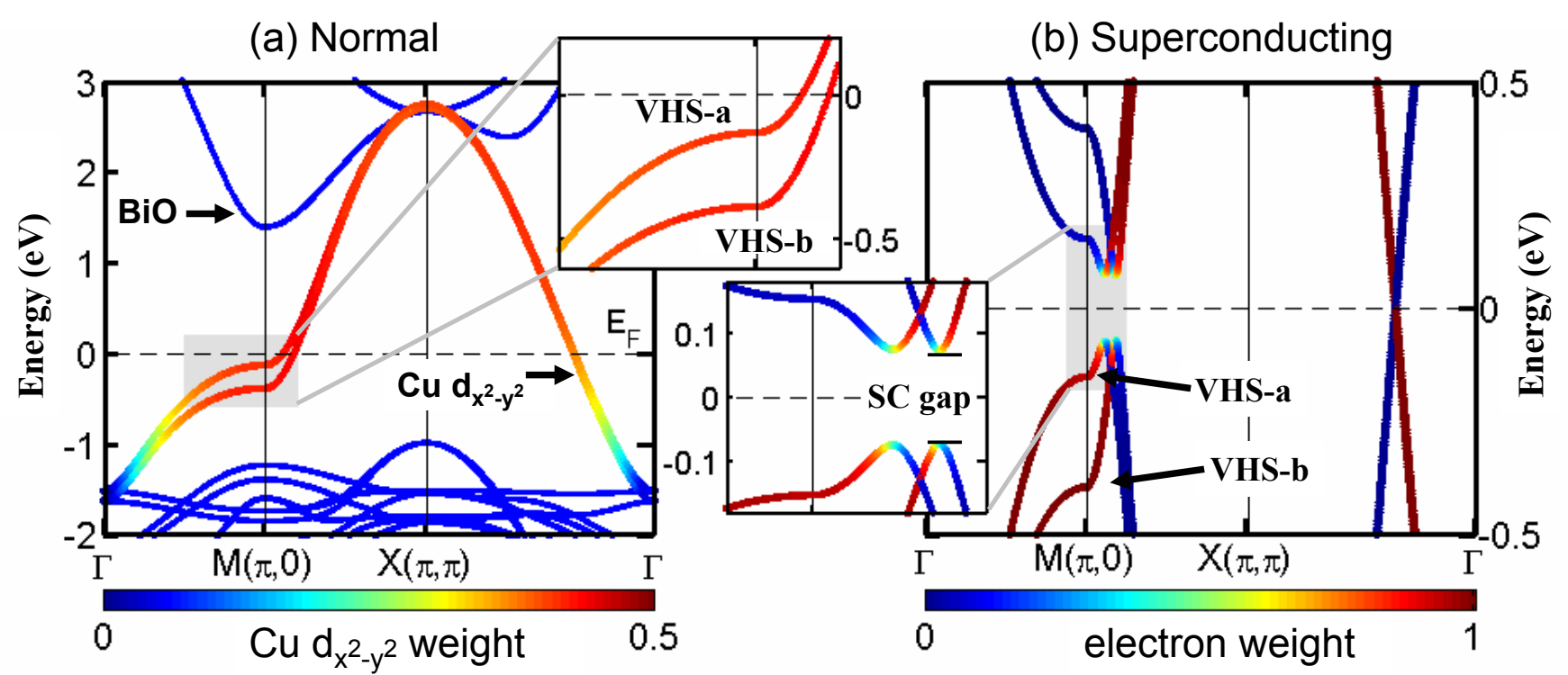

FIG. 2. (a) Normal state electronic spectrum of Bi2212. $\mathrm{Cu} d_{x^{2}-y^{2}}$ weight of states is given by the color of dots. Inset shows the energy region of the VHS's on an expanded scale. (b) Electronic spectrum of the superconducting state. Electron weight of states is given by the color of dots. Inset highlights the region of the superconducting gap near the antinodal point.

$\Delta_{k}=\frac{\Delta}{2}\left[\cos k_{x} a-\cos k_{y} a\right]$, where $a$ is the in-plane lattice constant. This interaction allows electrons of opposite spin to combine into superconducting pairs such that the resulting superconducting gap is zero along the nodal directions $k_{x}= \pm k_{y}$, and is maximum along the antinodal directions.

The Bi2212 sample is modeled as a slab of seven layers in which the topmost layer is $\mathrm{BiO}$, followed by layers of $\mathrm{SrO}, \mathrm{CuO}_{2}, \mathrm{Ca}, \mathrm{CuO}_{2}, \mathrm{SrO}$, and $\mathrm{BiO}$, as shown in Fig. 1(a). The tunneling computations are based on a $2 \sqrt{2} \times 2 \sqrt{2}$ real space supercell consisting of 8 primitive surface cells with a total of 120 atoms (see Fig. 1(b)). The coordinates are taken from the tetragonal crystal structure of Ref. 7, The tip-surface interaction is modeled by Slater-Koster overlap integrals between an s-orbital with a constant density of states and the surface orbitals in the vicinity of the tip. The tip is allowed to scan across the substrate to generate the topographic STM map, or held fixed on top of a surface Bi atom for the STS spectra.

The tight-binding parameters are fitted to the LDA band structure of Bi2212 that underlies for example the extensive angle-resolved photointensity computations of Ref. 8. The Slater-Koster results [9, 10] are used to fix the angular dependence of the tight binding overlap integrals. The specific orbital sets used for various atoms are: $\left(s, p_{x}, p_{y}, p_{z}\right)$ for $\mathrm{Bi}$ and $\mathrm{O} ; s$ for $\mathrm{Sr}$; and $\left(4 s, d_{3 z^{2}-r^{2}}, d_{x y}, d_{x z}, d_{y z}, d_{x^{2}-y^{2}}\right)$ for $\mathrm{Cu}$ atoms. This yields 58 orbitals in a primitive cell, used in band calculations, and a total of 464 orbitals for Green function supercell calculations in 256 evenly distributed k-points. Finally a gap parameter value of $|\Delta|=0.045 \mathrm{eV}$ is chosen to model a typical experimental spectrum [1] for the generic purposes of this study.

The LDOS and tunneling computations are based on Green function formalism. At first, the normal-state Green function is constructed via Dyson's equation using methodology described in Ref. 11. At this stage a selfenergy for orbital $\alpha, \Sigma_{\alpha}^{ \pm}=\Sigma_{\alpha}^{\prime} \pm i \Sigma^{\prime \prime}{ }_{\alpha}$ is embedded in Dyson's equation for possible effects of various bosonic couplings and correlation effects [12, 13]. For simplicity, we have assumed the self-energy to be diagonal in the chosen basis [14. In building up the Green function in the superconducting state, we utilize the conventional BCS-type self-energy $\Sigma^{B C S}=\Delta G^{h} \Delta^{\dagger}$ (see, e.g., Ref. 15), where $G^{h}$ is the hole part of normal state Green function.

Fig. 2 shows the calculated band structure of Bi2212 in the normal and the superconducting state from Hamiltonians of Eqs. 1 and 2. The normal state is seen to properly display the major features such as: The pair of $\mathrm{CuO}_{2}$ bands crossing the Fermi energy $\left(E_{F}\right)$ with the associated van Hove singularities (VHS's) marked VHS-a (antibonding) and VHS-b (bonding), split by $250 \mathrm{meV}$ at the $(\pi, 0)$ point; $\mathrm{BiO}$ bands lying about $1 \mathrm{eV}$ above $E_{F}$; and the 'spaghetti' of bands involving various $\mathrm{Cu}$ and $\mathrm{O}$ orbitals starting at a binding energy of around 1 $\mathrm{eV}$ below $E_{F}$. Although states near $E_{F}$ are mainly of $\mathrm{Cu} d_{x^{2}-y^{2}}$ and $\mathrm{O} p_{x, y}$ character, they also contain some $\mathrm{Bi}$ and $\mathrm{Cu} d_{z^{2}}$ admixture. In the superconducting state in Fig. 2(b), a quasiparticle spectrum mirrored through $E_{F}$ is obtained with a doubled number of bands due to the pairing interaction. A d-wave superconducting gap opens up in both $\mathrm{CuO}_{2}$ bands near $E_{F}$. The quasiparticles have a mixed electron/hole character only near the 

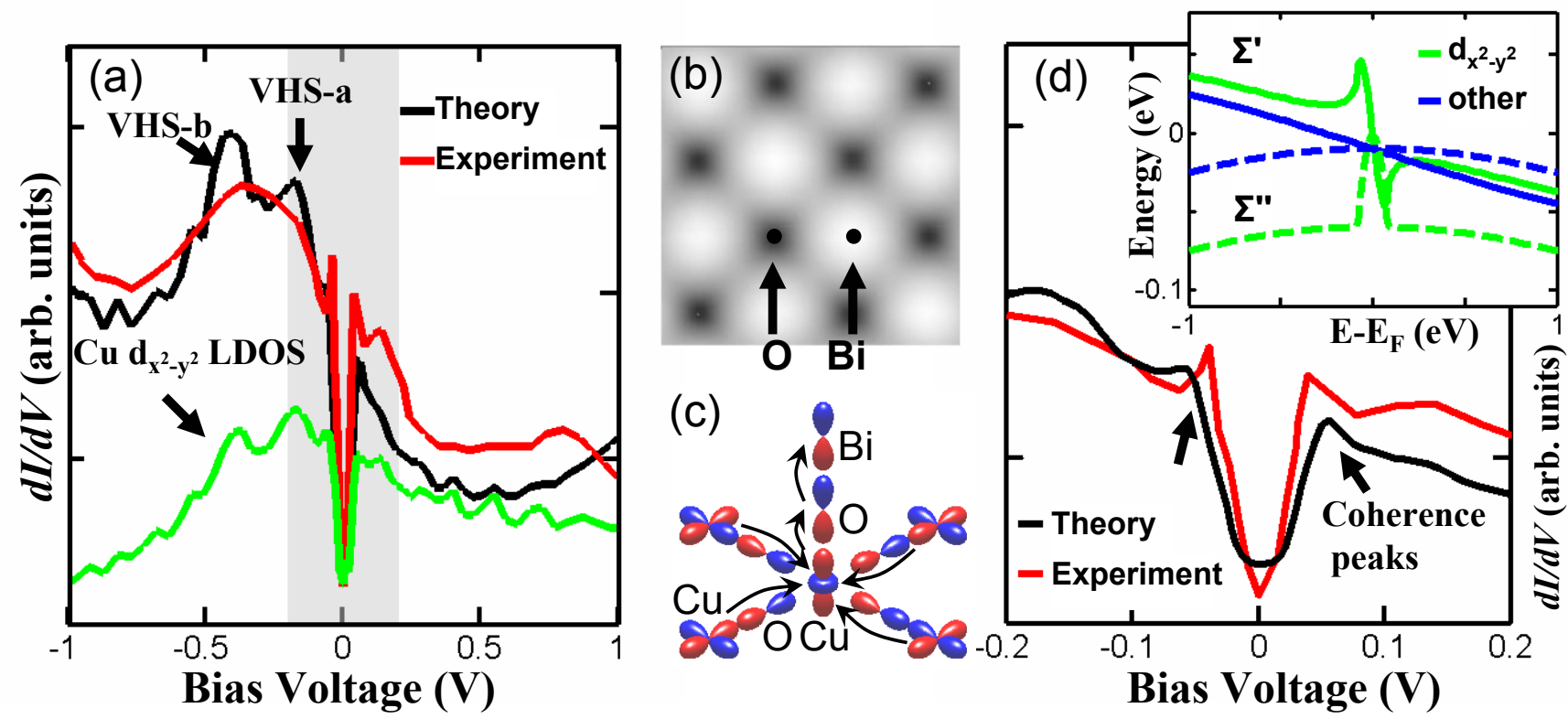

FIG. 3. (a) Computed (black line) and experimental (red line) differential tunneling spectra, $d I / d V$, as a function of bias voltage (in volts). LDOS of $\mathrm{Cu} d_{x^{2}-y^{2}}$ electrons is shown (green line) as a function of energy, where the horizontal scale is in $\mathrm{eV}$. (b) Computed topographic image of the $\mathrm{BiO}$ surface in which $\mathrm{Bi}$ atoms are bright and $\mathrm{O}$ atoms are dark. (c) The main tunneling channel from $\mathrm{Cu} d_{x^{2}-y^{2}}$ electrons on nearest neighbor $\mathrm{Cu}$ atoms to the tip through overlap with the central $\mathrm{Cu}$ atom, followed by hopping to the $p_{z}$ orbitals of the apical O-atom and the $\mathrm{Bi}$ atoms. (d) Computed and measured spectra in the low energy region marked by gray shading in (a). Inset gives the real (solid lines) and imaginary (dashed lines) parts of the self-energy applied to the $\mathrm{Cu} d_{x^{2}-y^{2}}$ states and to all other states [14].

edges of the gap.

To compute the tunneling spectra we apply the Todorov-Pendry expression [16, 17, for the differential conductance $\sigma$ between orbitals of the tip $\left(t, t^{\prime}\right)$ and the sample $\left(s, s^{\prime}\right)$, which in our case yields

$$
\sigma=\frac{d I}{d V}=\frac{2 \pi e}{\hbar} \sum_{t t^{\prime} s s^{\prime}} \rho_{t t^{\prime}}\left(E_{F}\right) V_{t^{\prime} s} \rho_{s s^{\prime}}\left(E_{F}+e V\right) V_{s^{\prime} t}^{\dagger},
$$

where the density matrix $\rho_{s s^{\prime}}=-\frac{1}{\pi} \sum_{\alpha} G_{s \alpha}^{+} \Sigma^{\prime \prime}{ }_{\alpha} G_{\alpha s^{\prime}}^{-}$ is, in fact, the spectral function written in terms of retarded/advanced Green function and the self-energy. Eq. 3 differs from the more commonly used TersoffHamann approach 18 in that it takes into account the details of the symmetry of the tip orbitals and their overlap with the surface orbitals.

The use of the spectral function recasts Eq. 3 into the form

$$
\sigma=\sum_{t \alpha} T_{t \alpha}
$$

where

$$
T_{t \alpha}=-\frac{2 e}{\hbar} \sum_{t^{\prime} s s^{\prime}} \rho_{t t^{\prime}}\left(E_{F}\right) V_{t^{\prime} s} G_{s \alpha}^{+} \Sigma^{\prime \prime}{ }_{\alpha} G_{\alpha s^{\prime}}^{-} V_{s^{\prime} t}^{\dagger},
$$

and the Green functions and the self-energy are evaluated at energy $E=E_{F}+e V_{b}$. Eq. 5 is similar to the
Landauer-Büttiker formula for tunneling across nanostructures (see, e.g., Ref. 19), and represents a slight reformulation of Refs. 20 and 21 .

The nature of Eq. 5 can be understood straightforwardly: $G_{s \alpha}$ gives the amplitude with which electrons residing on the $\alpha^{t h}$ orbital in the solid propagate to the surface at energy $E$ broadened by $\Sigma^{\prime \prime}{ }_{\alpha}$. The term $V_{s t}$ is the overlap between the surface orbital and the tip, while $\rho_{t t^{\prime}}$ gives the available states at the tip. Hence, $T_{t \alpha}$ gives the contribution of the $\alpha^{t h}$ orbital to the current, and the summation in Eq.4 4 collects these individual contributions to yield the total tunneling current which reaches the tip. Thus, selecting individual terms in Eq. 5 provides a transparent scheme to define tunneling paths between the sample and the microscope tip.

Fig. 3(a) shows the tunneling spectra over the broad energy range of $\pm 1 \mathrm{eV}$. At high positive voltages, the computed spectrum (black line) is fairly structureless. At low energies, a gap accompanied by the characteristic peak-dip-hump features is observed. The calculations show the anti-bonding (VHA-a) and bonding (VHS-b) VHS's 13 as distinct structures extending from -0.1 to $-0.5 \mathrm{eV}$, followed by a broad dip around $-0.7 \mathrm{eV}$ and subsequent rise near $-1 \mathrm{eV}[22,23$. In all these respects, the present computations substantially follow the experimental results (red line). 1 In particular, theory reproduces the observed asymmetry of the tunneling spectrum with excess intensity at negative biases. The rapid increase in 
current at high binding energies results from increasing spectral weight of $\mathrm{Cu} d_{z^{2}}$ and other orbitals contributing to the 'spaghetti' of bands starting around $1 \mathrm{eV}$ binding energy (see Fig. 2(a)). We emphasize that the LDOS of the $\mathrm{Cu} d_{x^{2}-y^{2}}$ (green line in Fig. 3(a)) does not provide a good description of the spectrum. In particular, the $\mathrm{Cu} d_{x^{2}-y^{2}}$ LDOS possesses an asymmetry which is opposite to that of the tunneling spectrum.

Fig. 3(d) gives a blow up of the low energy region of $\pm 0.2 \mathrm{eV}$, shown by gray shading in Fig. 3(a). The computed spectrum is seen to reproduce the coherence peaks and the characteristic peak-dip-hump feature. The generic form of the real and imaginary parts of the selfenergies applied to the $\mathrm{Cu} d_{x^{2}-y^{2}}$ orbitals (solid and dashed blue lines, respectively) and the rest of the orbitals are given in the inset 14. Fig. 3(b) shows the computed 'topographic map' of the $\mathrm{BiO}$ surface in constant current mode. Bi atoms appear as bright spots in accord with experimental observations, while $\mathrm{O}$ atoms sit at the centers of dark regions.

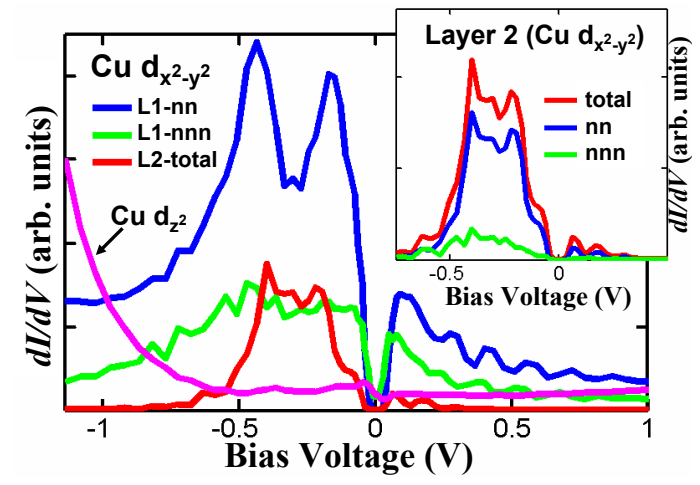

FIG. 4. Main frame: Partial contributions to the tunneling current from various orbitals in the two cuprate layers. The $\mathrm{CuO}_{2}$ layer closest to the tip is identified as layer 1 or L1, while the second layer is denoted by L2. Specific contributions are: $d_{x^{2}-y^{2}}$ orbitals of the four nearest neighbor $\mathrm{Cu}$ atoms (L1-nn, blue line); $d_{x^{2}-y^{2}}$ orbitals of the four next nearest neighbor $\mathrm{Cu}$ atoms of the first layer (L1-nnn, green line) ; $d_{x^{2}-y^{2}}$ orbitals of the $\mathrm{Cu}$ atoms of the second layer (L2, red line); $d_{z^{2}}$ of the central $\mathrm{Cu}$ atom of L1 (magenta line). Inset: Decomposition of the current from the second cuprate layer: Total contribution (red line); contribution of the four nearest neighbours (blue line); and the next nearest neighbours (green line).

An analysis of the partial terms of Eq. 5 reveals that the $d_{x^{2}-y^{2}}$ orbital of the $\mathrm{Cu}$ atom lying right under the $\mathrm{Bi}$ atom gives zero contribution to the current [24]. The dominant contribution to the spectrum comes from the four nearest neighbor $(\mathrm{NN}) \mathrm{Cu}$ atoms as indicated schematically in Fig. 3(c). A detailed decomposition of Eq. 4 is shown in Fig. 4, where paths starting from the $\mathrm{CuO}_{2}$ layer closest to the tip (L1), as well as from the second cuprate layer (L2) are considered. The signal from cuprate layers is dominated by the $d_{x^{2}-y^{2}}$ orbitals on the four nearest neighbour (nn) $\mathrm{Cu}$ atoms in L1 up to about $-0.7 \mathrm{eV}$ (blue line). At higher binding energies, the contribution from the $d_{z^{2}}$ electrons from the $\mathrm{Cu}$ atom right below the $\mathrm{Bi}$ atom or the tip grows rapidly (magenta line).

A smaller but still significant contribution comes from the four next nearest neighbour (nnn) $d_{x^{2}-y^{2}}$ orbitals in L1 spread over a wide energy range (green line, main figure), while the total current originating from the $d_{x^{2}-y^{2}}$ orbitals of L2 is quite localized over zero to $-0.6 \mathrm{eV}$ bias (red line, main figure). Fig. 4 emphasizes the nature of the current associated with the cuprate layers and points out an intrinsic electron-hole asymmetry originating from the $d_{z^{2}}$ orbitals. We note however that the $\mathrm{Bi}$ and $\mathrm{O}$ orbitals in the surface Bi-O layer can also play a role in producing an asymmetric background current.

In conclusion, we find that STS spectrum for Bi2212 is strongly modified from the LDOS of $d_{x^{2}-y^{2}}$ by the effect of the tunneling process or what we may call the tunneling matrix element. Much of the observed asymmetry of the spectrum can be explained within the conventional picture due to the turning on of $\mathrm{Cu} d_{z^{2}}$ and other channels with increasing (negative) bias voltage. This indicates that the effects of strong electronic correlations on the tunneling spectrum are more subtle than has been thought previously. However, we should note that we have not analyzed spectra associated with the deeply underdoped regime where charge order has been reported 25. The present method naturally allows an analysis of the tunneling signal in terms of the possible tunneling channels and the related selection rules. Our scheme can be extended to incorporate effects of impurities and various nanoscale inhomogeneities by using appropriately larger basis sets in the computations.

\section{Acknowledgments}

This work is supported by the US Department of Energy, Office of Science, Basic Energy Sciences contract DE-FG02-07ER46352, and benefited from the allocation of supercomputer time at NERSC, Northeastern University's Advanced Scientific Computation Center (ASCC), and the Institute of Advanced Computing, Tampere.

\footnotetext{
* jouko.nieminen@tut.fi

[1] K. McElroy et al., Science 309, 1048 (2005).

[2] E.W. Hudson et al., Nature 411, 920 (2001).

[3] A.N. Pasupathy et al., Science 320, 196 (2008).

[4] A.V. Balatsky et al., Rev. Mod. Phys. 78, 373 (2006).

[5] Ø. Fischer et al., Rev. Mod. Phys. 79, 353(2007).

[6] T. Hanaguri et al., Nature 430, 1001 (2004).
} 
[7] V. Bellini, F. Manghi, T. Thonhauser, and C. AmbroschDraxl, Phys. Rev. B 69, 184508(2004).

[8] H. Lin, S. Sahrakorpi, R.S. Markiewicz, and A. Bansil, Phys. Rev. Lett. 96, 097001 (2006).

[9] J.C. Slater and G.F. Koster, Phys. Rev. 94, 1498 (1954).

[10] W.A. Harrison, Electronic Structure and Properties of Solids. Dover, New York (1980).

[11] J.A. Nieminen and S. Paavilainen, Phys. Rev. B 60, 2921 (1999).

[12] B.W. Hoogenboom, C. Berthod, M. Peter, Ø. Fischer, and A.A. Kordyuk, Phys. Rev. B 67, 224502(2003).

[13] G. Levy de Castro et al., cond-mat/0703131 (2007).

[14] The specific self-energy used is shown in the inset to Fig. $3(d)$. This includes a Fermi liquid broadening $\left(E^{2}\right)$ of all the levels. The peak-dip-hump feature in the $d_{x^{2}-y^{2}}$ orbitals is modeled via coupling to a generic bosonic mode.

[15] A.L. Fetter and J.D. Walecka, Quantum Theory of ManyParticle Systems. Dover (2003).

[16] T.N. Todorov et al., J.Phys.: Condens. Matter 5, 2389 (1993).

[17] J.B. Pendry et al., J.Phys.: Condens. Matter 3, 4313
(1991).

[18] J. Tersoff and D.R. Hamann, Phys. Rev. B 31, 805 (1985).

[19] Y. Meir and N.S. Wingreen, Phys. Rev. Lett. 68, 2512 (1992).

[20] H. Ness and A.J. Fisher, Phys. Rev. B 56, 12469 (1997).

[21] T. Frederiksen, M. Paulsson, M. Brandbyge, and A.P. Jauho, Phys. Rev. B 75, 205413 (2007).

[22] The distinct VHS peaks in the computed spectrum are expected to be broadened to yield a broad hump much like the experimental spectrum due to self-energy corrections resulting from magnetic response of the electron gas in the $-400 \mathrm{meV}$ range (see, e.g., Ref. 23). These self-energy corrections are not included in the present calculations.

[23] R.S. Markiewicz, S. Sahrakorpi, and A. Bansil, Phys. Rev. B 76, 174514 (2007).

[24] I. Martin, A.V. Balatsky, and J. Zaanen, Phys. Rev. Lett. 88, 097003 (2002).

[25] Y. Kohsaka et al., Science 315, 1380 (2007). 